\title{
Sestrin modulator NV-5138 produces rapid antidepressant effects via direct mTORC1 activation
}

\author{
Taro Kato, ${ }^{1,2}$ Santosh Pothula, ${ }^{1}$ Rong-Jian Liu, ${ }^{1}$ Catharine H. Duman, ${ }^{1}$ Rosemarie Terwilliger, ${ }^{1}$ George P. Vlasuk, ${ }^{3}$ Eddine Saiah, ${ }^{3}$ \\ Seung Hahm, ${ }^{3}$ and Ronald S. Duman ${ }^{1}$ \\ 'Departments of Psychiatry and Neurosciences, Yale University School of Medicine, New Haven, Connecticut, USA. ${ }^{2}$ Sumitomo Dainippon Pharma Co., Osaka, Japan. ${ }^{3}$ Navitor Pharmaceuticals Inc., Cambridge, \\ Massachusetts, USA.
}

\begin{abstract}
Preclinical studies demonstrate that rapid-acting antidepressants, including ketamine, require stimulation of mTORC1 signaling. This pathway is regulated by neuronal activity and endocrine and metabolic signals, notably including the amino acid leucine, which activates mTORC1 signaling via binding to the upstream regulator sestrin. Here, we examined the antidepressant actions of NV-5138, a highly selective small molecule modulator of sestrin that penetrates the blood-brain barrier. The results demonstrate that a single dose of NV-5138 produced rapid and long-lasting antidepressant effects and rapidly reversed anhedonia caused by chronic stress exposure. The antidepressant actions of NV-5138 required brain-derived neurotrophic factor (BDNF) release, as the behavioral responses were blocked by infusion of a BDNF-neutralizing Ab into the medial prefrontal cortex (mPFC) or, in mice, with a knockin of a BDNF polymorphism that blocked activity-dependent BDNF release. NV-5138 administration also rapidly increased synapse number and function in the $\mathrm{mPFC}$ and reversed the synaptic deficits caused by chronic stress. Together, the results demonstrate that NV-5138 produces rapid synaptic and antidepressant behavioral responses via activation of the mTORC1 pathway and BDNF signaling, indicating that pharmacological modulation of sestrin may be an attractive approach for the development of rapid-acting antidepressants.
\end{abstract}

\section{Introduction}

Major depressive disorder (MDD) is a chronic, debilitating illness that affects approximately $17 \%$ of the population and is one of the leading causes of disability worldwide (1). Currently, MDD is typically treated with drugs that influence monoamine neurotransmission, but large-scale clinical trials (i.e., $\mathrm{STAR}^{*} \mathrm{D}$ ) show that these drugs can take weeks to months to produce a therapeutic response, have limited efficacy, and result in low rates of full remission (24). On the other hand, recent studies demonstrate that a single, subanesthetic dose of ketamine produces rapid (within hours) and long-lasting (7 to 10 days) antidepressant effects even in patients who have failed to respond to traditional antidepressants and are considered treatment resistant $(5,6)$.

Although the exact mechanisms underlying the antidepressant actions of ketamine are not fully known, preclinical studies demonstrate a key role for mTORC1 signaling and increased synapse formation in the medial prefrontal cortex (mPFC) $(7,8)$. Ketamine rapidly stimulates mTORC1 signaling in the $\mathrm{MPFC}$, and infusion of rapamycin, a selective mTORC1 inhibitor, into the mPFC blocks the synaptic and antidepressant behavioral actions

\section{Related Commentary: p. 2207}

Conflict of interest: This work was funded by Navitor Pharmaceuticals. RSD has consulted and/or received research support from Naurex, Allergan, Lilly, Forest, Johnson \& Johnson, Taisho Pharmaceutical Co., Sunovion, and Navitor. Copyright: () 2019, American Society for Clinical Investigation. Submitted: December 17, 2018; Accepted: April 2, 2019; Published: May 20, 2019. Reference information: J Clin Invest. 2019;129(6):2542-2554. https://doi.org/10.1172/JCl126859. of ketamine $(7,8)$. A single dose of ketamine also rapidly increases synapse number and function in the $\mathrm{MPFC}$ and reverses the synaptic deficits caused by chronic stress exposure $(7,8)$. A similar role for mTORC1 signaling has been demonstrated in the synaptic and behavioral responses to several other rapid-acting antidepressants tested, including the GluN2B allosteric modulator Ro 25-6981 $(7,8)$, the mGluR2/3 antagonist LY341495 (9), the metabolite (2R,6R)-hydroxynorketamine (10), the glycine-like partial agonist rapastinel (11), and the muscarinic antagonist scopolamine (12). In addition, viral expression of a constitutively active form of p70S6 kinase, a downstream mTORC1 effector, is sufficient to produce an antidepressant response (13). These studies indicate that mTORC1 signaling is necessary and may be sufficient to produce rapid synaptic and antidepressant behavioral responses.

The mTORC1 signaling pathway regulates cellular protein synthesis and serves as a key sensor of cellular activity, hormonal signals, and nutrient levels (14-16). Amino acids are the primary macromolecular components of proteins, and rates of protein synthesis are regulated by their availability. Leucine is one of the key regulatory amino acids and can promote protein synthesis in large part through regulation of mTORC1 signaling (17-19). Recent studies demonstrate that leucine binds to sestrin isoforms 1 and 2 (hereafter referred to as sestrin), resulting in dissociation from another regulatory multiprotein complex, GATOR2, leading to activation of mTORC1 signaling $(20,21)$. Further evidence for this pathway is provided by studies demonstrating that mutations of Sestrin 2 that block leucine binding also block leucine activation of mTORC1 signaling $(20,21)$.

This leucine-sensing pathway provides an approach for manipulating mTORC1 signaling and determining whether acti- 
vation of this pathway produces rapid antidepressant responses. NV-5138 was designed as a synthetic leucine analogue that readily penetrates the blood-brain barrier and selectively binds sestrin to activate mTORC1 signaling in the brain (22). In addition, NV-5138 is highly selective for sestrin without modulation of other CNS targets, including binding to or functional modulation of NMDA receptors (22). NV-5138 is not a substrate for the key leucine metabolizing pathways and is not incorporated into proteins (22). These unique features of the compound result in rapid induction of mTORC1 activity in the brain via sestrin modulation. This contrasts with leucine, which is rapidly metabolized and incorporated into proteins, resulting in relatively constant levels that are insensitive to dietary augmentation (23). These features, coupled with a short pharmacokinetic half-life of roughly 3 hours in the rat brain and peripheral circulation, result in rapid, but transient, activation of mTORC1 (22). Here, we show that NV-5138 produces rapid synaptic changes in rodent $\mathrm{mPFC}$ and antidepressant behavioral responses that are similar to those that result from ketamine, but without NMDA receptor modulation, supporting a key role for mTORC1 signaling and providing a target for antidepressant drug development.

\section{Results}

NV-5138 produces ketamine-like antidepressant behavioral responses. The effects of NV-5138 were tested in standard behavioral paradigms that are responsive to antidepressants, including tests of behavioral despair (forced swim test [FST]) and anxiety (novelty suppressed feeding test [NSFT]). In the first study, rats received oral administration of vehicle, NV-5138 (40, 80, or $160 \mathrm{mg} / \mathrm{kg}$, p.o.) or leucine $(160 \mathrm{mg} / \mathrm{kg}$, p.o.) and behavioral testing started 24 hours later (Figure 1A). In the FST and NSFT, analyzed 1 or 3 days after dosing, NV-5138 significantly reduced the immobility time and latency to feed, respectively, at $160 \mathrm{mg} / \mathrm{kg}$, but not at 40 or $80 \mathrm{mg} / \mathrm{kg}$ (Figure 1, B and D). This dose response is consistent with doses of NV-5138 required to activate mTORC1 signaling in the brain (22). There was no effect of leucine $(160 \mathrm{mg} / \mathrm{kg})$ in these paradigms, including the FST and NSFT (Figure 1, B and D). NV-5138 had no effect on locomotor activity or home-cage feeding $(\mathrm{HCF})$, indicating that the effects were specific for behavioral despair in the FST and anxiety in the NSFT and not due to general effects on ambulation or feeding (Figure 1, C and E).

Next, we conducted a female urine sniffing test (FUST), a paradigm used to assess motivation and reward as well as NSFT after NV-5138 (160 mg/kg, p.o.) and compared the response to that seen with the rapid acting antidepressant ketamine $(10 \mathrm{mg} /$ kg, i.p.) (Figure 1F). Previous studies demonstrate that this is an effective dose of ketamine for producing antidepressant behavioral responses in naive rodents and in a chronic stress model (7, 8). NV-5138 administration produced a significant increase in time sniffing female urine in the FUST and decreased latency to feed in the NSFT; these responses were comparable to those seen with ketamine (Figure 1, G and I). There were no effects of either NV-5138 or ketamine on locomotor activity, time spent sniffing water, or HCF (Figure 1, G, H, and J).

NV-5183 produces long-lasting antidepressant actions, similar to those of ketamine. Clinical findings demonstrate that ketamine causes long-lasting (7 to 10 days) as well as rapid antidepressant responses in depressed patients $(5,6)$; similar long-lasting effects have been observed in rodent models (24). To test the duration of the antidepressant action of NV-5138, rats were administered vehicle or NV-5138 (160 mg/kg, p.o.) or vehicle or ketamine (10 $\mathrm{mg} / \mathrm{kg}$, i.p.) and behavioral testing started 3 and 7 days later (Figure 2A). Both NV-5138 and ketamine showed significant reduction in immobility times 3 and 7 days after administration in the FST (Figure 2, B and D); there were no effects on latency to feed 10 days after administration in the NSFT (Figure 2, C and E). There were no effects of either NV-5138 or ketamine on HCF (data not shown).

Repeated low-dose NV-5138 $(80 \mathrm{mg} / \mathrm{kg})$ also produces antidepressant effects. The antidepressant effects of lower doses of NV-5138 (40 or $80 \mathrm{mg} / \mathrm{kg}$, p.o.) administered daily for a total of 7 days (starting with day 0) were also tested (Figure 2F). Because the antidepressant actions of ketamine begin to reverse after 7 days (24), ketamine (10 mg/kg, i.p.) was administered every other day for 6 days as a positive control. The results demonstrate that 80 $\mathrm{mg} / \mathrm{kg}$ of NV-5138 showed antidepressant effects by significantly reducing the immobility time and latency to feed in both the FST and NSFT, respectively,without alteration of locomotor activity or HCF (Figure 2, G-J). This was in contrast with the lack of efficacy at this dose when evaluated 24 hours following a single administration (Figure 1, B and D). Ketamine administration also produced significant effects on immobility time and latency to feed in the FST and NSFT, respectively (Figure 2, G and J).

NV-5138 rapidly reverses the behavioral and synaptic deficits caused by chronic stress. The development of anhedonia, a core symptom of depression, with chronic unpredictable stress (CUS) exposure and the requirement for chronic treatment of a typical antidepressant to reverse this effect makes CUS one of the most valid models of depression $(25,26)$. The CUS model also provides a rigorous test for fast-acting antidepressants, and a single dose of ketamine rapidly reverses CUS-induced anhedonia, determined in a sucrose-preference test (SPT) (8). The results of the current study demonstrate that repeated CUS exposure (21 days) decreases sucrose preference and that a single dose of NV-5138 rapidly reverses this effect (Figure 3, A and B). NV-5138 administration did not influence sucrose preference in nonstressed control rats (Figure 3B). There was no significant difference in the total amount of water or total volume of liquid consumed (Supplemental Figure 1, $\mathrm{A}$ and B; supplemental material available online with this article; https://doi.org/10.1172/JCI126859DS1). CUS exposure was continued for subsequent behavioral testing and tissue sampling on day 26. CUS exposure increased latency to feed in the NSFT (day 22), and NV-5138 rapidly reversed this effect (Figure 3C). There were no effects of CUS or NV-5138 on HCF conducted immediately following the NSFT (Figure 3D), indicating that the effects of NV-5138 were not due to general increases in feeding. CUS exposure significantly decreased body weight, a predicted outcome of chronic stress exposure (Figure 3E).

Chronic stress paradigms also profoundly alter brain structure and function in rodents, causing reductions in levels of synapseassociated proteins in the $\mathrm{MPFC}$ as well as a reduction in spine synapse number (25-32). Studies were conducted to determine whether NV-5138 reverses these synaptic protein changes. For this study, rats were exposed to an additional 5 days of CUS after 
$\mathbf{A}$

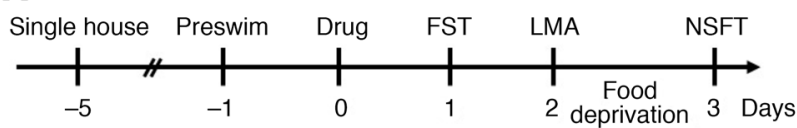

B

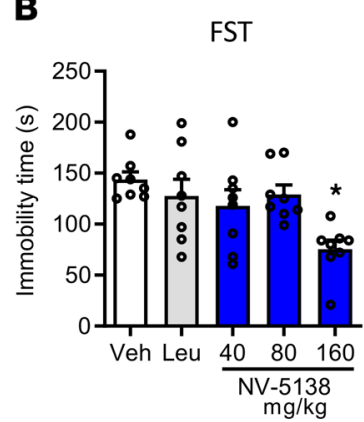

c

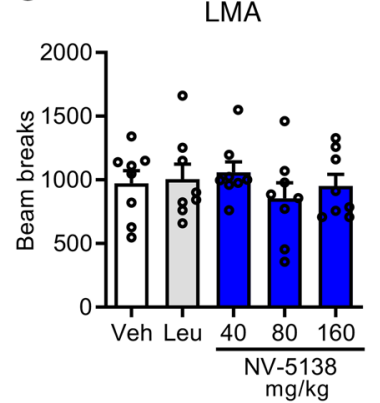

D

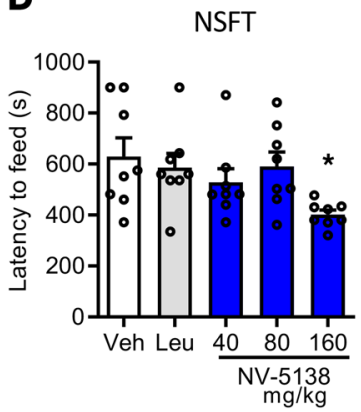

E

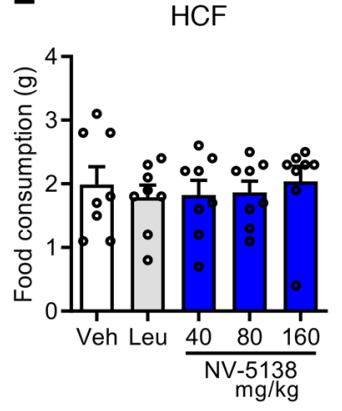

$\mathbf{F}$

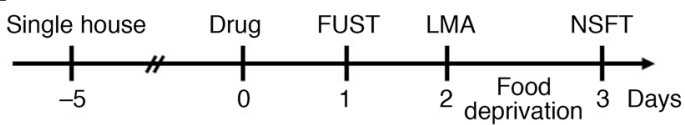

G

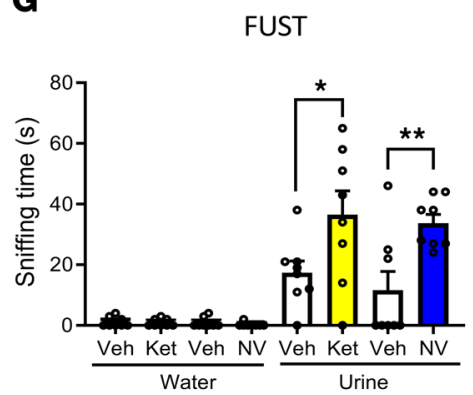

H

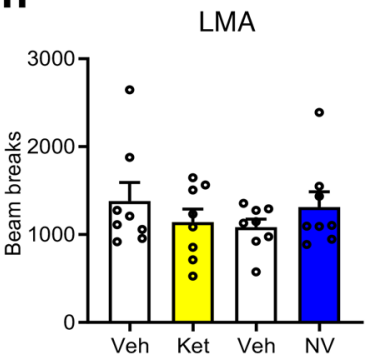

I

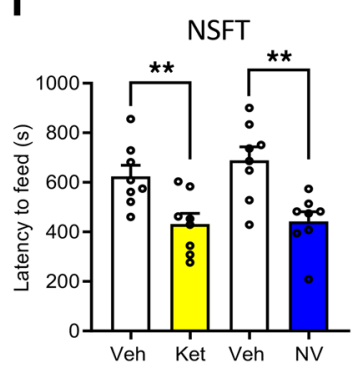

J

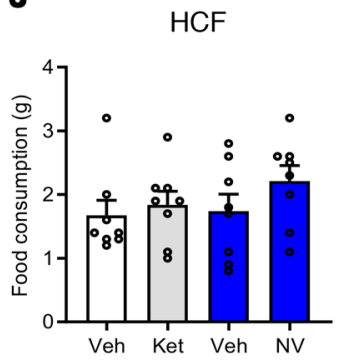

Figure 1. NV-5138 shows ketamine-like antidepressant actions in rodent models of depression. (A) Beginning 24 hours after NV-5138 (40, 80 , or 160 mg/ $\mathrm{kg} \mathrm{p.o.)} \mathrm{administration,} \mathrm{behavioral} \mathrm{studies} \mathrm{were} \mathrm{conducted} \mathrm{over} 3$ consecutive days (B-E). NV-5138 treatment significantly decreased immobility time and latency to feed at $160 \mathrm{mg} / \mathrm{kg}$ in $(B)$ the FST $\left(F_{4,35}=3.24, P<0.01\right)$ and $(D)$ the NSFT $\left(F_{4,35}=2.67, P<0.05\right)$, respectively. No significant effects were seen in (C) locomotor activity $\left(F_{4,35}=0.536, P>0.05\right)$ or $(E) H C F\left(F_{4,35}=0.223, P>0.05\right)$. (F) Beginning 24 hours after ketamine $(10 \mathrm{mg} / \mathrm{kg}$ i.p.) or NV-5138 (160 mg/ $\mathrm{kg}, \mathrm{p.o.}$ ) administration, behavioral studies were conducted over the next 3 days (G-J). Both ketamine and NV-5138 significantly increased female urine sniffing time and decreased latency to feed in (G) the FUST and (I) the NSFT, respectively. No significant effects were seen in (H) locomotor activity or (J) HCF. The results are shown as mean \pm SEM. $n=8$ /group. ${ }^{*} P<0.05$; ${ }^{* *} P<0.01$, Tukey's multiple comparison test, following significant results of 1-way ANOVA (B-E, H-J) or Student's $t$ test (G). Veh, vehicle; Leu, leucine; Ket, ketamine; NV, NV-5138; LMA, locomotor activity test.

the initiation of behavioral studies (Figure 3A). CUS exposure decreased levels of GluR1 and PSD95 in synaptosome preparations of $\mathrm{mPFC}$, and a single dose of NV-5138 reversed these synaptic protein deficits (Figure 3, F and $\mathrm{G}$ ).

NV-5138-induced antidepressant actions require mTORC1 signaling. The effects of NV-5138 on the phosphorylated and activated forms of several mTORC1-signaling proteins in the $\mathrm{mPFC}$ were determined 1 hour after dosing (Figure 4, A and B). As expected, a single dose of NV-5138 $(160 \mathrm{mg} / \mathrm{kg})$ rapidly increased levels of phospho-mTOR as well as the downstream targets, phospho-p70S6K1, and phospho-4EB-P1, in synaptosomal preparations of PFC (Figure 4, C-E), confirming recent studies (22). A single dose of ketamine $(10 \mathrm{mg} / \mathrm{kg}$ ) produced effects (Figure 4, C-E) similar to those previously reported $(7,8,11,24)$.

To determine whether the behavioral actions of NV-5138, like ketamine and other rapid-acting antidepressants, require mTORC1 signaling, the selective mTORC1 inhibitor rapamycin was infused into the mPFC 30 minutes before NV-5138 (Figure
4F). The cannula placements and infusion sites are shown in Figure 4G. NV-5138 was administered (160 mg/kg), and 24 hours later, behavior was tested (Figure $4 \mathrm{~F}$ ). Note that the preswim was inadvertently conducted 4 hours after NV-5138 administration, which differed from the typical paradigm of 24 hours before dosing. However, NV-5138 still produced a robust antidepressant response by significantly reducing the immobility time and latency to feed in the FST and NSFT, respectively, with no effect on HCF or locomotor activity (Figure 4, H-K). Prior infusion of rapamycin into $\mathrm{mPFC}$ completely blocked the behavioral actions (reduction in immobility time and latency to feed) of NV-5138 in both FST and NSFT (Figure 4, $\mathrm{H}$ and J). Previous studies demonstrate that infusion of rapamycin alone into the $\mathrm{MPFC}$ has no effect on these behaviors $(7,8,10,12)$.

Antidepressant actions of NV-5138 require BDNF. Previous studies demonstrate that the antidepressant actions of ketamine and other rapid acting antidepressant including scopolamine and rapastinel require brain-derived neurotrophic factor (BDNF), 
A

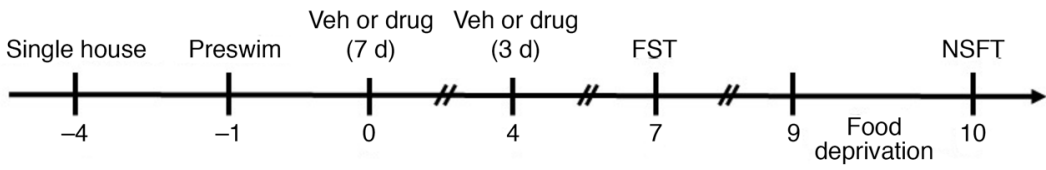

B

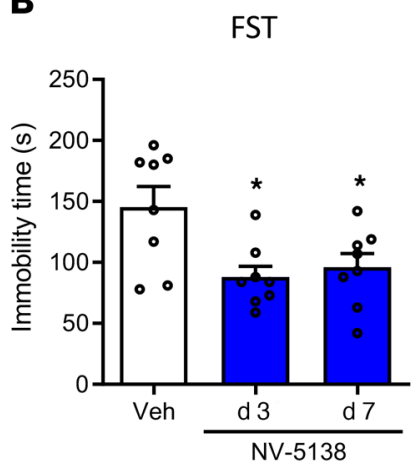

C

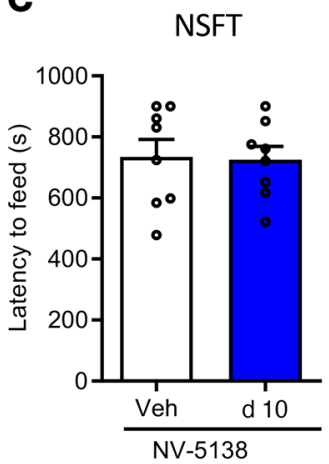

D

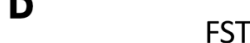

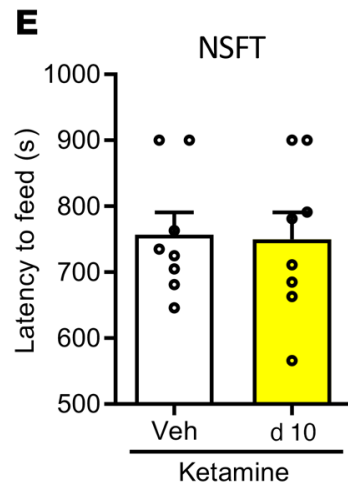

F

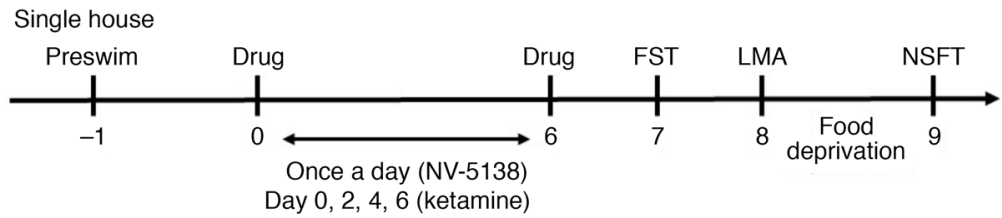

G

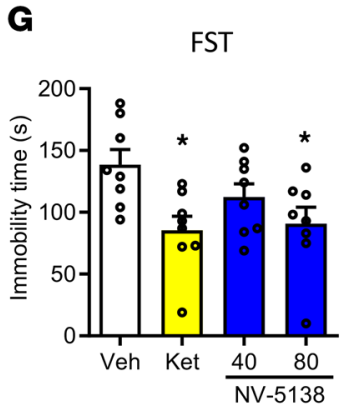

H

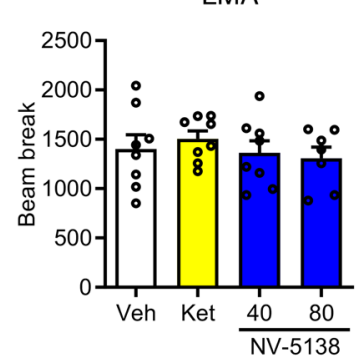

I

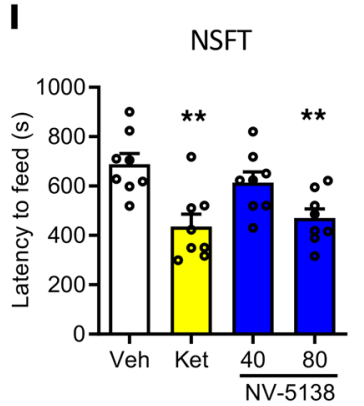

J

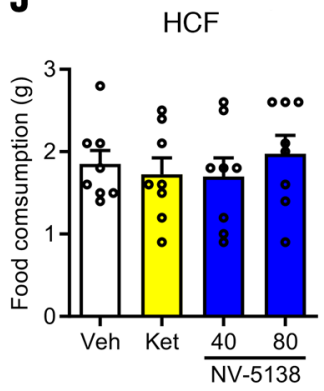

Figure 2. Single-dose NV-5183 produces long-lasting antidepressant effects, and repeated low-dose NV-5138 also produces antidepressant effects. (A) Three or seven days after NV-5138 $(160 \mathrm{mg} / \mathrm{kg})$ or ketamine $(10 \mathrm{mg} / \mathrm{kg})$ administration, the FST was conducted. Three days after the FST, the NSFT was conducted. Both NV-5138 and ketamine significantly decreased immobility time in the FST 3 and 7 days after administration (B and $\mathbf{D})\left(\mathrm{NV}-5138 ; \mathrm{F}_{221}=\right.$ $5.82, P<0.01$, ketamine; $\left.F_{2,21}=5.47, P<0.05\right)$ but had no effect on latency to feed in the NSFT on day 10 ( $C$ and $\mathbf{E}$ ). (F) Low-dose NV-5138 was administered once a day for 7 days, and ketamine was administered on alternate days over the same time frame. Twenty-four hours after the last administration, behavioral studies were conducted over the next 3 days (G-J). Both ketamine and $80 \mathrm{mg} / \mathrm{kg}$ of NV-5138 significantly decreased immobility time in the FST (I) $\left(F_{3,28}=4.05, P<0.05\right)$ and latency to feed in the NSFT $(K)\left(F_{3,28}=7.29, P<0.001\right)$. No significant effects were seen in $(H)$ locomotor activity $\left(F_{3,27}=0.500\right.$ $P>0.05)$ or $(\mathrm{J}) \mathrm{HCF}\left(\mathrm{F}_{3,28}=0.380, P>0.05\right)$. The results are shown as mean \pm SEM. $n=8$ /group. ${ }^{*} P<0.05 ;{ }^{* *} P<0.01$, Tukey's multiple comparison test, following significant results of 1-way ANOVA (B, D, G-J) or Student's $t$ test (C and $\mathbf{E})$.

determined by infusion of a BDNF neutralizing $\mathrm{Ab}(\mathrm{nAb})$ or in BDNF mutant mice (33-38). Here, we evaluated the role of BDNF in the antidepressant actions of NV-5138 using both of these approaches. Rats received intra-mPFC infusion of a BDNF (nAb) $(0.5 \mu \mathrm{g} / \mathrm{side}) 30$ minutes before NV-5138 $(160 \mathrm{mg} / \mathrm{kg}$ p.o.) and behavioral testing started 24 hours later (Figure $5 \mathrm{~A}$ ). In control IgG-infused rats, NV-5138 produced significant reduction in immobility time and latency to feed in the FST and NSFT, respectively, but BDNF nAb infusion completely blocked these effects (Figure 5, B and D); there were no effects of BDNF nAb alone in the FST or NSFT or on locomotor activity or HCF (Figure 5, B-E).

The BDNF Met polymorphism blocks the processing and activity-dependent release of mature BDNF (33). Here, the behavioral actions of NV-5138 were examined in BDNF Val66Met knockin mice. WT Val/Val littermates, Val/Met heterozygous, and Met/Met knockin mice were examined in antidepressant behavioral models according to the schedule in Figure $5 F$. There were significant genotype effects between $\mathrm{Val} / \mathrm{Val}$ and Met/Met littermates in the FST or NSFT (Figure 5, G and I). In WT mice, we observed significant reduction in immobility time and latency to feed upon NV-5138 treatment in both the FST and NSFT, respectively, with no effects on locomotor activity or HCF (Figure 5, H-J). The antidepressant actions of NV-5138 (reduction in immobility time and latency to feed) were completely blocked in the Val/Met and Met/Met mice in both the FST and NSFT (Figure 5, G and I). 
A

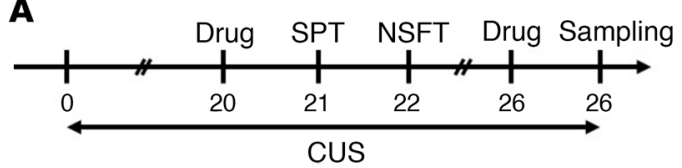

B

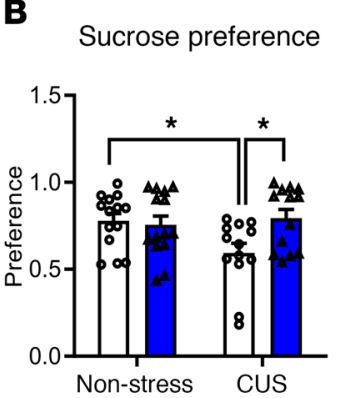

C

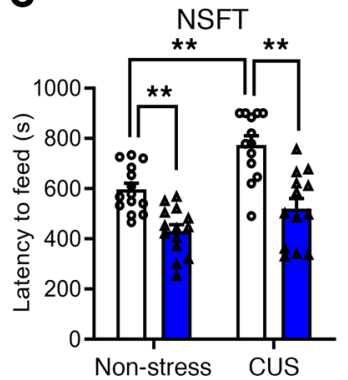

D

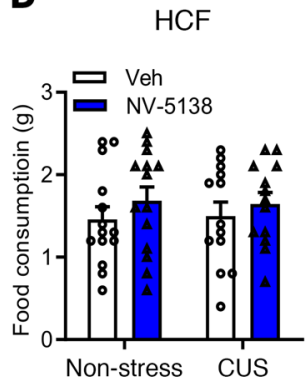

$\mathbf{F}$

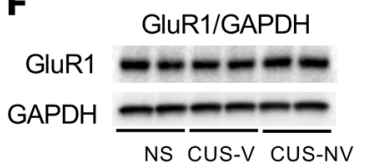

G
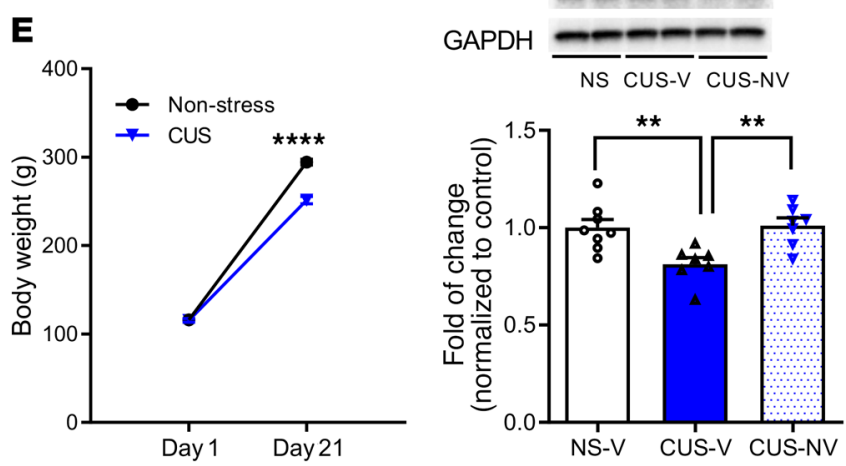

Figure 3. NV-5138 reverses the behavioral and synaptic deficits caused by CUS. (A) Schematic for CUS experiment and effect of NV-5138 ( $160 \mathrm{mg} / \mathrm{kg}$, day 20 of CUS) on the SPT and NSFT (on days 21 and 22 of CUS). CUS was continued throughout the behavioral testing and tissue sampling on day 26 . After a short washout period, a second dose of NV-5138 was administered on CUS day 25 and tissue was collected on day 26. (B-D) Results are the mean \pm SEM. $n=13-14 /$ group. (B) CUS decreased sucrose preference and NV-5138 reversed this effect; there was a significant interaction between CUS and NV-5138 administration $\left(F_{1,50}=5.20, P<0.05\right.$, effect of CUS: $\mathrm{F}_{150}=2.21, P>0.05$, effect of NV-5138: $\mathrm{F}_{150}=3.27, P>0.05$ ). Also, a 3-way ANOVA analysis revealed a significant interaction among CUS, NV-5138 administration, and sucrose consumption $\left(F_{1,50}=4.02, P=0.05\right)$. (C) CUS increased the latency to feed in NSFT, and NV-5138 reversed this effect $\left(F_{1,50}=1.89, P>0.05\right.$, effect of CUS: $F_{1,50}=17.52, P<0.001$, effect of NV-5138: $\left.F_{1,50}=43.4, P<0.0001\right)$. (D) There was no significant effect on HCF $\left(F_{1,50}=0.0679, P>0.05\right.$. (E) CUS significantly decreased body weight $\left(F_{1,104}=47.06, P<0.0001\right.$, effect of day: $F_{1,104}=2612, P<0.0001$, effect of CUS: $\left.F_{1,50}=49.86, P<0.0001\right)$. Results are shown as mean \pm SEM. $n=13-14$ / group. ${ }^{*} P<0.05$; ${ }^{*} P<0.01$, 2-way ANOVA and Tukey's multiple comparison test. ( $\mathbf{F}$ and $\mathbf{G}$ ) CUS decreased levels of the postsynaptic proteins GluR1 and PSD95 in PFC, and NV-5138 reversed these deficits (GluR1, $F_{2,19}=$ 7.95, $P<0.01$; PSD95, $\left.F_{2,19}=6.31, P<0.01\right)$. Results are shown as mean \pm SEM. $n=7-8 /$ group. ${ }^{*} P<0.05 ;{ }^{* *} P<$ $0.01,{ }^{* * * *} P<0.001,1$-way ANOVA (B-D, F, G) and Student's $t$ test (E). NS, NSFT; V, vehicle.

NV-5138 increases synaptic number and function in mPFC pyramidal neurons. Previous studies demonstrate that a single dose of ketamine increases spine synapse number as well as function (i.e., increased frequency of 5-HT and hypocretin-induced EPSCs) of layer $\mathrm{V}$ pyramidal neurons in the $\mathrm{mPFC}(7,8,10)$. Using the same approach, whole-cell patch recordings of layer $\mathrm{V}$ pyramidal cells, the influence of NV-5138 on 5-HT- and hypocretin-induced EPSCs in layer $\mathrm{V}$ neurons was determined at submaximal concentrations (20 $\mu \mathrm{M}$ and $200 \mathrm{nM}$, respectively). Twenty-four hours following a single dose of NV-5138, the frequency of hypocretin-induced EPSCs was significantly increased and the increase of 5-HTinduced EPSCs approached significance $(P=0.052)$ (Figure 6, A
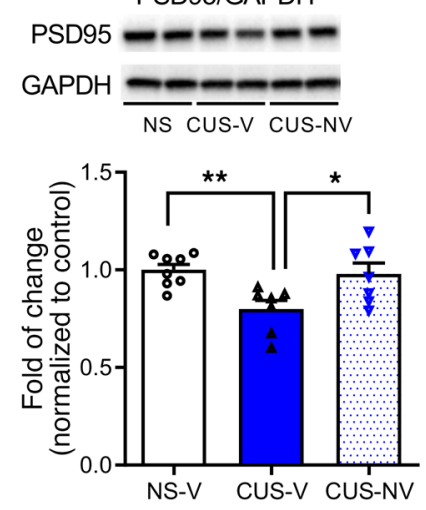

and B). NV-5138 also significantly increased the amplitudes of both hypocretin and 5-HT-induced EPSCs, as shown in the cumulative fraction graphs (Supplemental Figure 2A).

Layer $\mathrm{V}$ pyramidal neurons were passively filled with neurobiotin during recording to allow for subsequent imaging and analysis of dendritic spines. Dendritic segments in the apical tuft of filled neurons were scanned using confocal laser microscopy. In NV-5138-treated rats, spine density was significantly increased in the layer $\mathrm{V}$ pyramidal cells due to significantly increased densities of thin and mushroom spines (Figure 6, C and D). Similar effects on spine density have been observed with ketamine $(7,8)$. There were no significant effects on overall spine-head diameter (see frequency distribution analysis, Supplemental Figure 2B). Levels of synaptic proteins were also examined 24 hours after a single dose of NV-5138 or ketamine. Both agents significantly increased levels of GluR1, synapsin 1, and SV2A; only ketamine significantly increased levels of PSD95, although there was a trend following NV-5138 (Figure 6, E-H). There were no significant differences in levels of GAPDH, which was used for normalization (Supplemental Figure 3). We have recently reported that NV-5138 significantly increases PSD95 in naive animals, confirming the trend observed in the current study (22).

Influence of AMPA receptor blockade on the antidepressant actions of NV-5138. Previous studies demonstrated that the actions of rapid-acting antidepressants require glutamate AMPA receptor activity, as pretreatment with the AMPA receptor antagonist NBQX blocks the antidepressant behavioral responses to ketamine, rapastinel, LY314597, (2R,6R)-HNK, and scopolamine (7, 10, 12, 39, 40). Here, we show that pretreatment (30 minutes) with NBQX (10 mg/ kg i.p.) (Figure 7A) did not significantly influence the antidepressant actions of NV-5138 on immobility time in the FST measured 24 hours after dosing (Figure 7B); there was a partial blockade of the NV-5138 response on latency to feed in the NSFT measured 3 days after dosing (Figure 7D). There were no significant effects of NBQX on locomotor activity or HCF (Figure 7, C and E). 
A

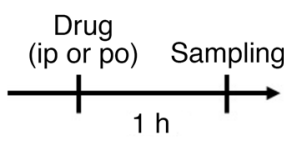

B

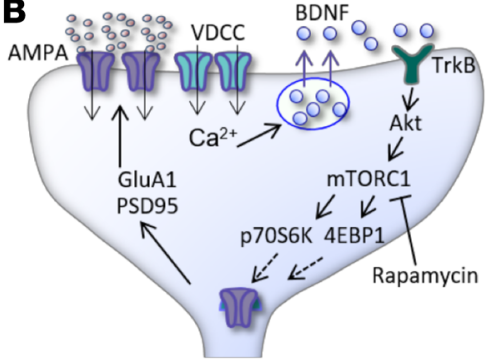

C

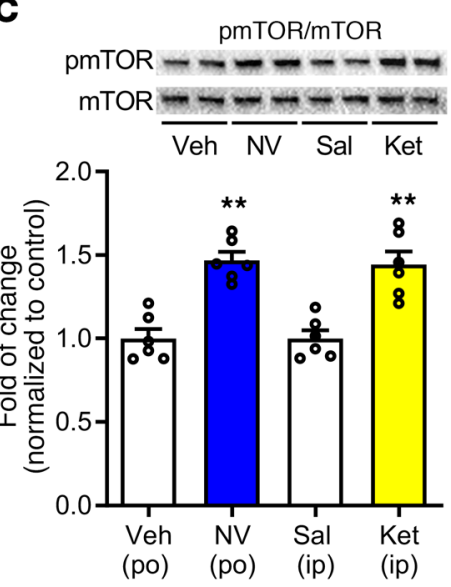

D
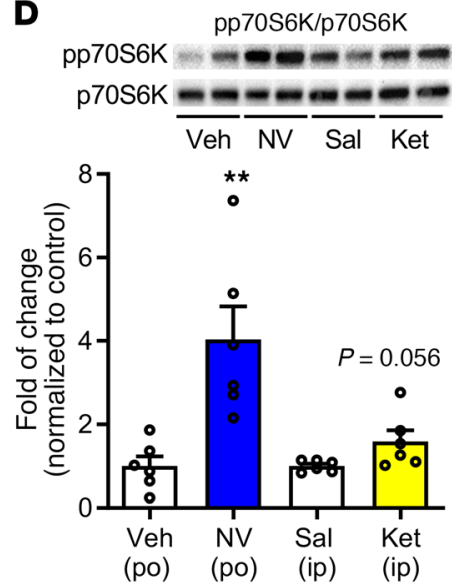

E
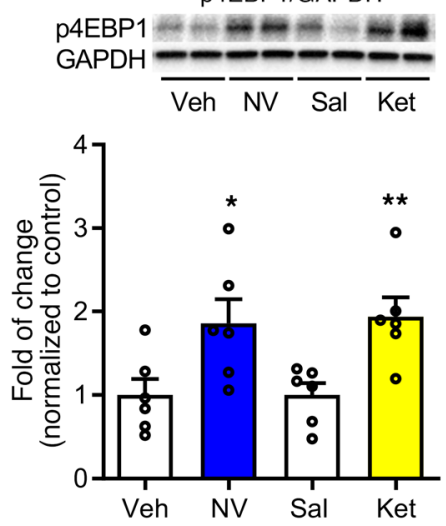

$\begin{array}{llll}\text { (po) (po) (ip) (ip) } & \end{array}$

$\mathbf{F}$
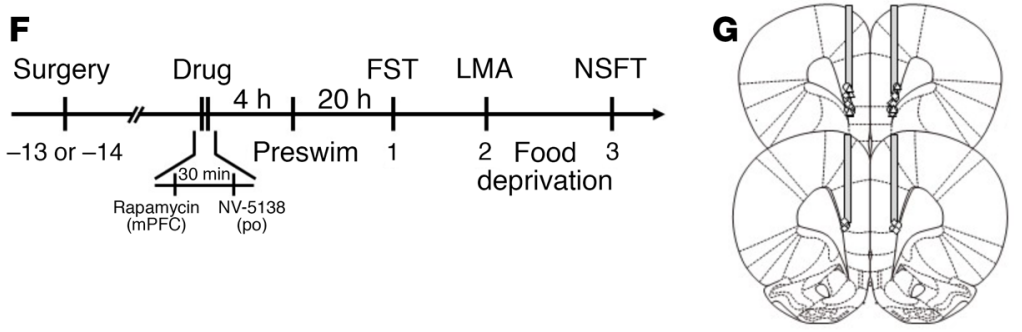

OVehicle/vehicle

$\diamond$ Vehicle/NV-5138

$\triangle \mathrm{Rapa} / \mathrm{NV}-5138$

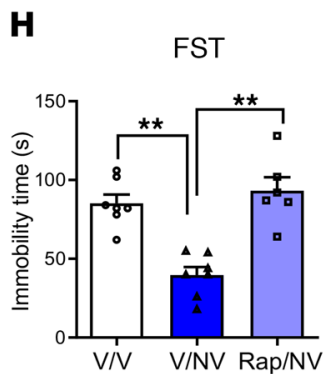

\section{I}

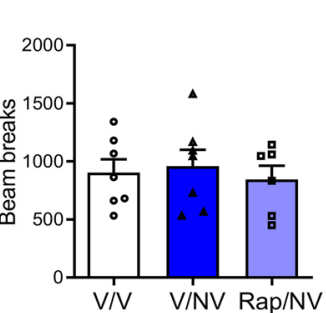

J

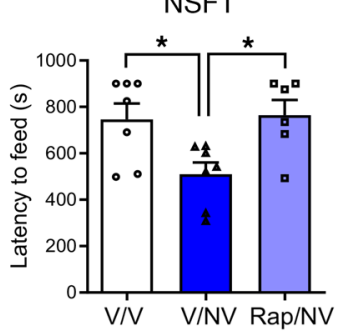

$\mathbf{K}$

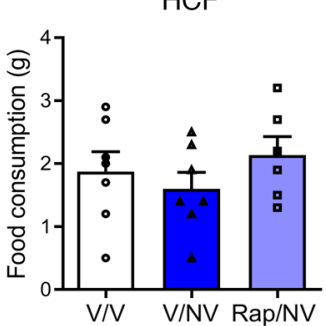

Figure 4. Antidepressant actions of NV-5138 are dependent on activation of mTORC1 signaling. (A) Rats were administered vehicle or NV-5138 (160 mg/ $\mathrm{kg}$ ) and PFC dissections were collected 1 hour later. (B) Diagram showing postsynaptic signaling. Levels of the phosphorylated and activated forms of (C) mTOR, (D) p70S6K, and (E) 4EBP1 as determined by Western blot analysis were increased by NV-5138 and ketamine; levels of total proteins or GAPDH were also measured to control for loading differences. Results are shown as mean \pm SEM. $n=6 /$ group. ${ }^{*} P<0.05$; ${ }^{* *} P<0.01$, Student's $t$ test. (F) Rats were implanted with bilateral cannula in the MPFC and allowed to recover for approximately 2 weeks. (G) The mTORC1 inhibitor rapamycin was infused into the mPFC 30 minutes prior to administration of vehicle or NV-5138. Twenty-four hours after NV-5138 administration, behavioral studies were initiated and conducted over the next 3 days (H-K). NV-5138 treatment significantly decreased immobility time and latency to feed, but these effects were blocked by rapamycin in $(\mathbf{H})$ the FST $\left(F_{2,17}=20.46, P<0.001\right)$ and $(J)$ the NSFT $\left(F_{2,17}=5.36, P<0.05\right)$, respectively. No significant effects were seen in $(I) L M A\left(F_{2,17}=\right.$ $0.200, P>0.05)$ or $(K) H C F\left(F_{2,17}=0.814, P>0.05\right)$. Results are shown as mean $\pm \mathrm{SEM} . n=6-7 .{ }^{*} P<0.05 ;{ }^{* *} P<0.01$, Tukey's multiple comparisons test, following significant results of 1-way ANOVA. Rap, rapamycin.

\section{Discussion}

The results demonstrate that a single dose of the mTORC1 activator NV-5138 produces rapid antidepressant actions in behavioral models of depression and treatment response. In addition, a single dose of NV-5138 increases the number and function of synapses and increases levels of synaptic proteins in the $\mathrm{MPFC}$. The results also demonstrate that these actions of NV-5138 require mTORC1 signaling as well as BDNF. Together, these findings demonstrate a mech- 
A

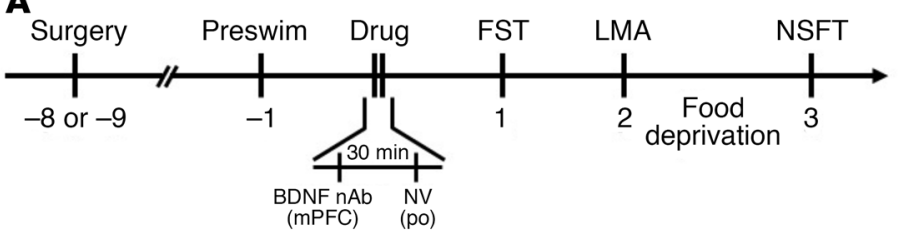

B

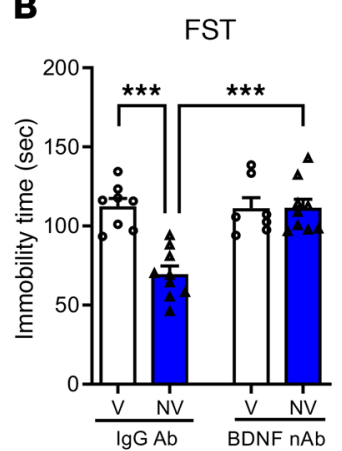

C

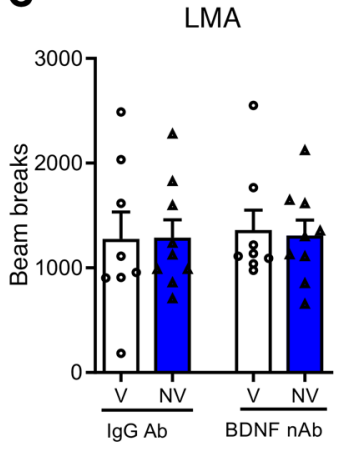

D

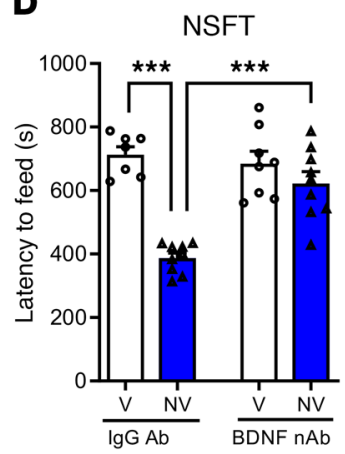

E

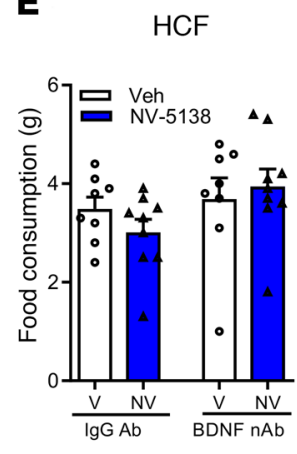

F

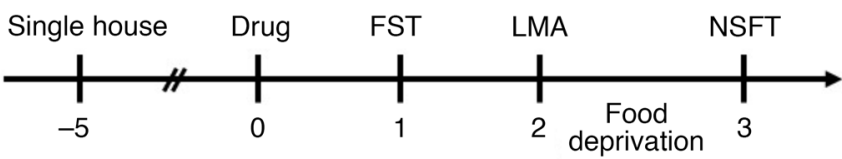

G

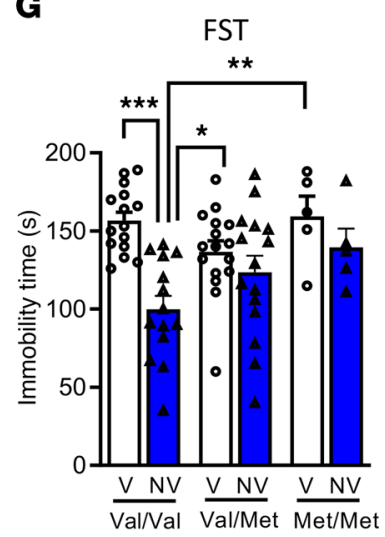

H

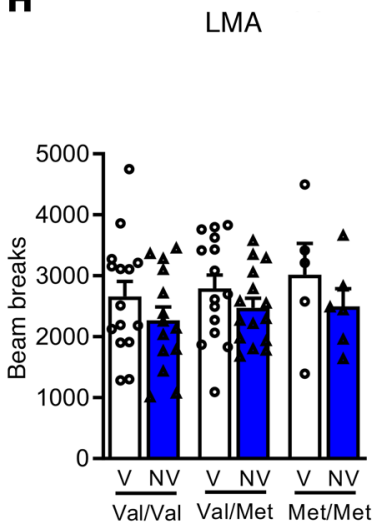

I

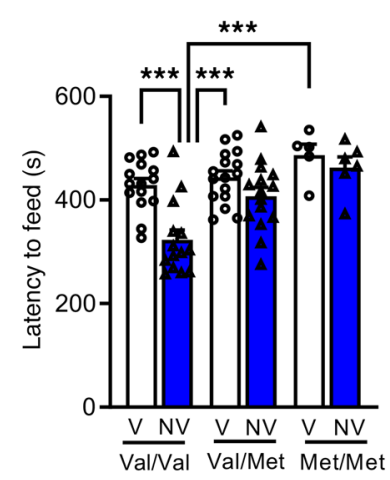

J

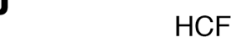

Figure 5. BDNF is required for antidepressant actions of NV-5138. (A-E) The antidepressant actions of NV-5138 are blocked by infusion of BDNF $n A b$ into the mPFC. (A) BDNF nAb or control lgG (0.5 $\mathrm{g} / \mathrm{side})$ was infused into the mPFC 30 minutes prior to administration of vehicle or NV-5138 (160 mg/kg, p.0.). (B and D) NV-5138 significantly decreased immobility time (B) in the FST (effect of NV-5138: $F_{1,29}=14.6, P<0.001$; mPFC infusion: $F_{1,29}=13.4, P<0.001$; interaction: $F_{1,29}=14.9, P<0.001$ ) and latency to feed (D) in the NSFT (effect of NV-5138: $F_{1,29}=39.4, P<0.001$; mPFC infusion: $F_{1,29}=11.2, P=0.002$; interaction: $F_{1,29}=18.0, P<0.001$ ), and these effects were blocked by infusion of the BDNF nAb. (C and $\mathbf{E}$ ) There were no significant effects on locomotor activity (effect of NV-5138: $\mathrm{F}_{1,30}=0.01, P=0.92$; mPFC infusion: $\mathrm{F}_{1,30}=0.07, P=0.79$; interaction: $\mathrm{F}_{1,30}=0.03, P=0.86$ ) or HCF (effect of NV-5138: $\mathrm{F}_{1,30}=0.11, P=0.74 ; \mathrm{mPFC}$ infusion: $\mathrm{F}_{1,30}=2.93, P=0.1$; interaction: $\left.\mathrm{F}_{1,30}=1.23, P=0.28\right)$. $(F-J)$ The antidepressant actions of NV-5138 are attenuated in BDNF Val66Met knockin mice. (F) Experimental time line for behavioral testing of animals after vehicle or NV-5138 administration. In the BDNF Val/Val mice, NV-5138 treatment significantly decreased immobility time (C) in the FST (effect of NV-5138: $F_{1.64}=12.7, P<0.001$; genotype: $F_{2.64}=1.88, P=0.16$; interaction: $F_{2.64}=3.98, P=0.02$ ) and latency to feed (I) in the NSFT (effect of NV-5138: $F_{1,65}=13, P<0.001$; genotype: $F_{2,65}=12.8, P<0.001$; interaction: $F_{2,65}=3.4, P=0.04$ ) that were blocked in $\mathrm{Val} /$ Met and Met/Met mice. No significant effects were observed in $(\mathbf{H})$ LMA (effect of NV-5138: $\mathrm{F}_{1,64}=3.39, P>0.05$; genotype: $F_{2,64}=0.59, P=0.56$; interaction: $\mathrm{F}_{2,64}=0.06, P=0.94$ ) or (J) HCF (effect of NV-5138: $\mathrm{F}_{1,65}=0.065, P=0.80$; genotype: $\mathrm{F}_{2,65}=0.52, P=0.60$; interaction: $\mathrm{F}_{2,65}=0.58, P=0.56$ ). Results are shown as mean \pm SEM. $n=7$-9/group $(\mathbf{A}-\mathbf{E}) ; n=5$-16/group $(\mathbf{F}-\mathrm{J})$. ${ }^{*} P<0.05 ;{ }^{* *} P<0.01 ;{ }^{* * *} P<0.001$ (A-J), 2-way ANOVA and Tukey's post hoc test.

anism, direct activation of $\mathrm{mTORC1}$ signaling via the upstream regulator sestrin (22), and are consistent with an mTORC1 requirement for the actions of other rapid antidepressant agents $(7,8,10,12)$.

The results demonstrate that a single oral dose of NV-5138 produces rapid antidepressant actions in 3 standard models of behavioral despair (FST), anxiety (NSFT), and motivation/reward
(FUST); these effects were dose dependent and were not associated with general effects on locomotor activity or HCF. In addition, the antidepressant actions of a single dose of NV-5138 persisted for up to 7 days in the FST. The FST is typically used for drug screening, but is responsive to acute administration of typical antidepressants (41); the NSFT is responsive to chronic ( $\sim 3$ weeks) administration of 


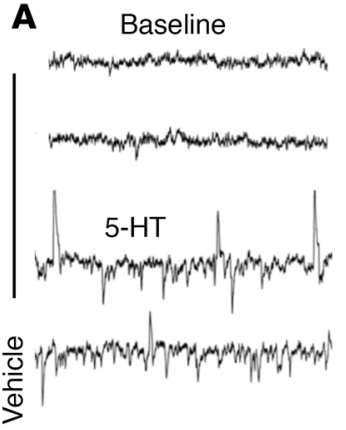

Hert

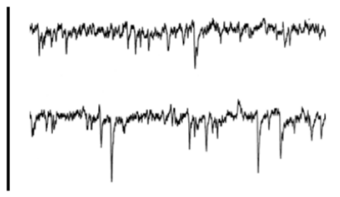

C
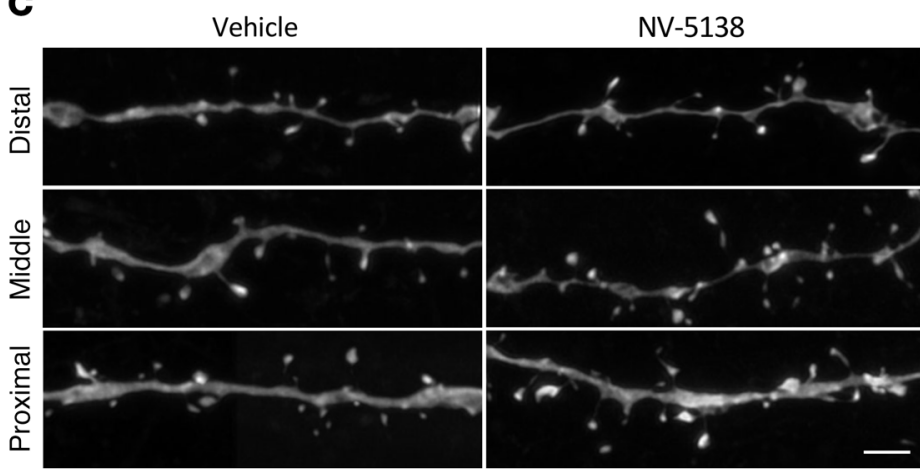

E

GluR1/GAPDH

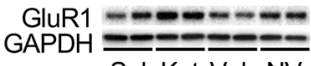

Sal Ket Veh NV

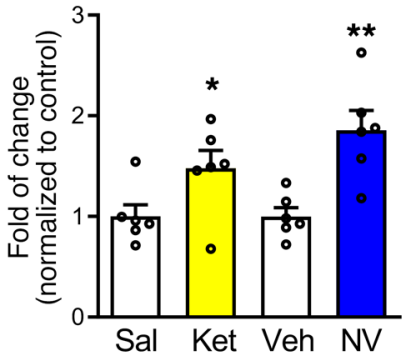

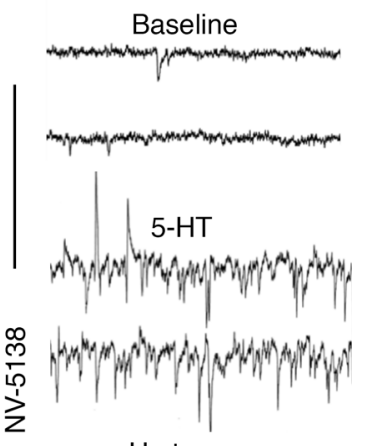

Hert
F

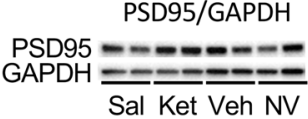

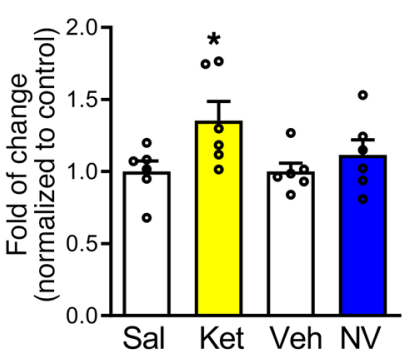

B

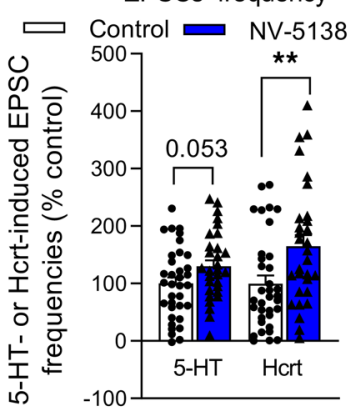

D

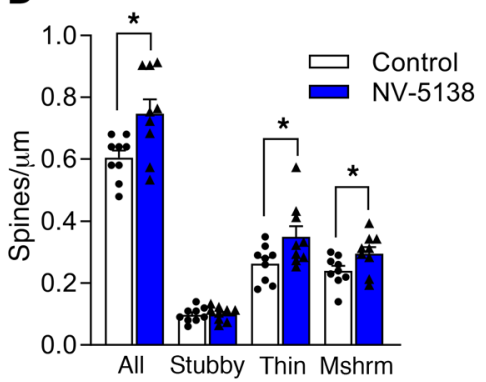

G

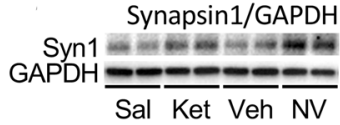

H

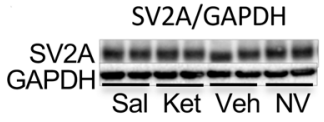

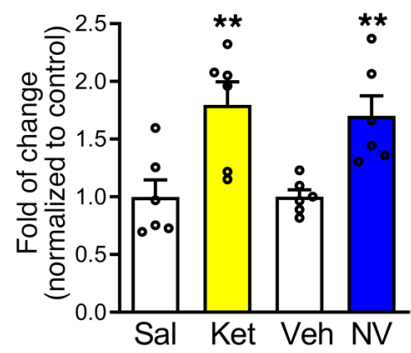

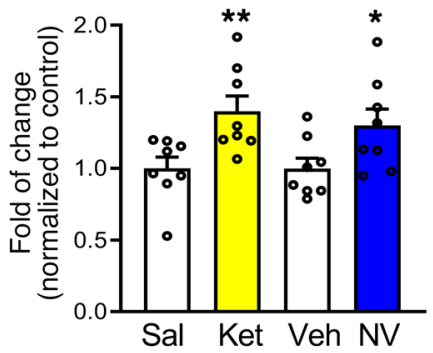

Figure 6. Influence of NV-5138 on spine number and function and synaptic proteins in the PFC. (A) Representative traces showing postsynaptic currents recorded from layer V pyramidal neurons in mPFC brain slices from vehicle- or NV5138-treated rats (24 hours after drug treatment). (B) Summary of data showing that frequencies of 5-HT- $(20 \mu \mathrm{M})$ and hypocretin-induced ( $200 \mathrm{nM})$ EPSCs are increased by NV-5138; $n=35$ cells $/ 8$ rats for control; $n=30$ cells $/ 8$ rats for NV-5138. ${ }^{*} P<0.05 ;{ }^{* *} P<0.01, t$ test. Absolute values for baseline EPSC frequency is $3.58 \pm 0.4(\mathrm{~Hz}) ; n=35$ cells/8 rats for control and $2.63 \pm 0.48$ $(\mathrm{Hz}) ; n=30$ cells/8 rats for NV-1538 (C). Representative images of high-magnification Z-stack projections of apical dendritic segments from slices collected 24 hours following vehicle or NV-5138 administration. Scale bar: $5 \mu \mathrm{m}$. (D) NV-5138 increased overall spine density $(t=2.72, P=0.015)$ due to increases in densities of thin $(t=2.20, P=0.04)$ and mushroom spines $(t=2.01, P=0.05) . n=9$ cells $/ 8$ rats for control and 9 cells $/ 5$ rats for NV-5138. (E-C) Rats were administered vehicle, ketamine $(10 \mathrm{mg} / \mathrm{kg})$, or NV-5138 $(160 \mathrm{mg} / \mathrm{kg})$, and PFC dissections were collected 24 hours later. Levels of the postsynaptic proteins including (E) GluR1, (F) PSD95, (G) synapsin1, and (H) SV2A were determined by Western blot analysis. GAPDH levels were also determined to control for loading differences. $n=6$ /group. ${ }^{*} P \leq 0.05 ;{ }^{* *} P<0.01$ Student's $t$ test.

typical antidepressants, but is a model of anxiety $(42,43)$. To further test the rapid antidepressant actions of NV-5138, we used a CUS model that results in anhedonia, a core symptom of depression that is only reversed by chronic administration of typical antidepressants $(25,26,44)$. The results show that a single dose of NV-5138 reverses the CUS-induced effects on sucrose preference as well as latency to feed in the NSFT. NV-5138, like other antidepressants, does not have an effect on sucrose preference in 
$\mathbf{A}$

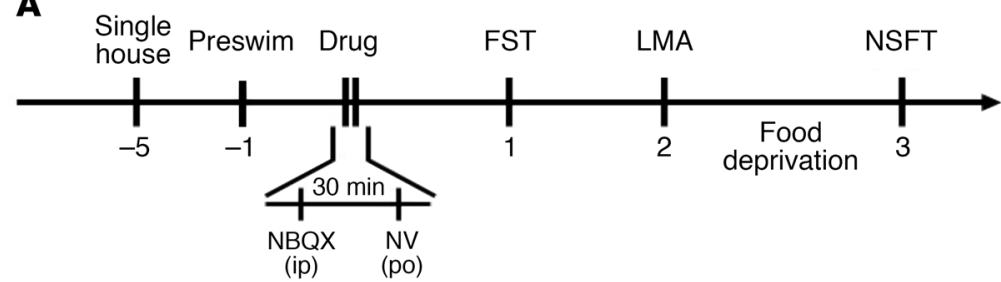

B

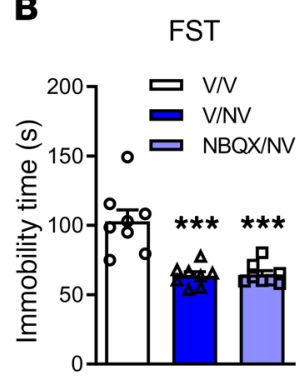

c

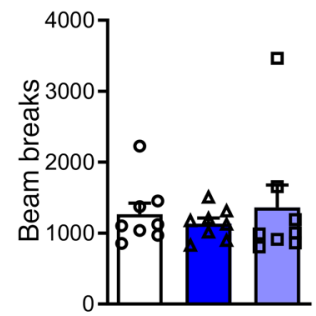

D

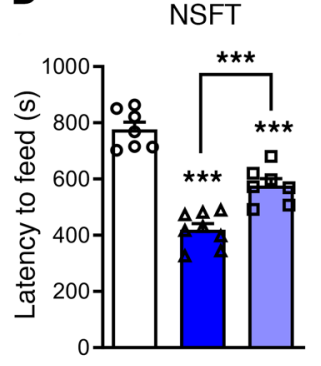

$\mathbf{E}$

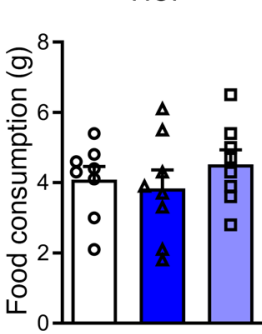

Figure 7. AMPA receptor antagonist NBQX does not block the antidepressant actions of NV-5138. (A) Rats were administered either vehicle (0.5\% DMSO, $1 \mathrm{ml} / \mathrm{kg}$, i.p.) or NBQX (10 mg/kg, i.p.) 30 minutes prior to administration of vehicle or NV-5138 (160 mg/kg, p.o.). Twenty-four hours after NV-5138 administration, behavioral studies were initiated and performed over the next 3 days. (B and $\mathbf{D})$ NV-5138 treatment significantly decreased immobility time (B) and latency to feed (D), but these effects were not blocked by pretreatment with NBQX in the FST (effect of treatment: $F_{2,21}=18.5, P<0.001$ ) and NSFT (effect of treatment: $F_{2,19}=56.5, P<0.001$ ), respectively. ( $C$ and $\mathbf{E}$ ) No significant effects were observed in LMA (effect of treatment: $F_{2,21}=0.304, P>0.05$ ) or HCF (effect of treatment: $F_{2,21}=0.625, P>0.05$ ). Results are shown as mean \pm SEM. $n=7$-8/group. ${ }^{* * *} P<0.001$ compared with control group, 1 -way ANOVA followed by post hoc Tukey's multiple comparison test.

naive, nonstressed rats due to a ceiling effect, but does influence latency to feed in the NSFT in both naive and nonstressed rats due to the innate fear of a novel environment even in naive rodents. Together, the results demonstrate that NV-5138 produces rapid antidepressant actions in 4 different behavioral models, similar to the effects of the prototypical rapid-acting agent ketamine $(7$, 8). The results are consistent with the hypothesis that NV-5138 produces antidepressant actions via stimulation of MTORC1 signaling, although this conclusion is tempered for the CUS study, since we did test the influence of NV-5138 on mTORC1 signaling in CUS-exposed rats.

We also found, as expected, that the antidepressant behavioral actions of NV-5138 were blocked by $\mathrm{mPFC}$ infusion of rapamycin, a selective mTORC1 inhibitor, consistent with evidence of increased mTORC1 signaling in the MPFC. We also examined the role of BDNF, since previous studies report that the rapid synaptic and antidepressant behavioral actions of ketamine are dependent on neuronal activity and require BDNF signaling (7, $34,37,39,45)$. Here, we show that the antidepressant actions of NV-5138 are also dependent on BDNF signaling using 2 different approaches. First, we show that the behavioral actions of NV-5138 in the FST and NSFT are blocked by MPFC infusion of a BDNF Ab that binds and neutralizes BDNF that is released into the interstitial space. Second, we show that the antidepressant actions of NV-5138 are completely blocked in mice with a knockin of the BDNF Met allele, which blocks the processing and activitydependent release of BDNF (33). This was observed in both Val/ Met and Met/Met knockin mice, similar to complete blockade of the antidepressant actions of ketamine and scopolamine in Met/ Met mice, and partial or complete block, respectively, in Val/Met mice $(37,38)$. These findings demonstrate that a single WT Val allele is not sufficient to support the actions of NV-5138, indicating that a full complement of mature BDNF is required. Together, the results demonstrate that the behavioral actions of NV-5138 are dependent on BDNF activity.

Fast-acting antidepressants such as ketamine also produce rapid synaptic changes in the MPFC that are associated with the antidepressant behavioral actions of these agents (45). Moreover, ketamine and other fast-acting agents rapidly reverse the synaptic deficits caused by chronic stress exposure and by depression, demonstrating that agents such as ketamine can rapidly reverse the pathophysiology of depression $(8,46)$. Here, the results demonstrate that a single dose of NV-5138 also rapidly increases the number and function of spine synapses in layer $\mathrm{V}$ pyramidal neurons in the MPFC. This includes increased hypocretin-induced EPSCs and a strong trend for increased 5-HT-induced EPSCs. NV-5138 also increased the number of spines on apical dendrites of layer $\mathrm{V}$ pyramidal neurons and increased levels of the synaptic proteins GluA1, synapsin 1, and SV2A. Moreover, in animals exposed to CUS, which caused a reduction in sucrose preference, there was a marked decrease in levels of the synaptic proteins GluA1 and PSD95 and these deficits were reversed by a single dose of NV-5138. Previous studies demonstrate that the ability of ketamine to increase synapse number and function are dependent on mTORC1 signaling $(7,8)$. Together, these findings demonstrate that NV-5138, like ketamine, can rapidly increase synapse number and function and reverse the effects of chronic stress via stimulation of mTORC1 signaling and synaptic protein synthesis.

Previous studies have demonstrated that MTORC1 signaling is required for the rapid behavioral actions of ketamine as well as other rapid-acting antidepressants. This information inspired the development of an agent that activates mTORC1 signaling via the 
target sestrin2. The current study demonstrates that the mTORC1 activator NV-5138 produces rapid synaptic and antidepressant behavioral responses and rapidly reverses the synaptic and behavioral deficits caused by chronic stress. These synaptic and behavioral responses are similar to those found with ketamine, but occur via an initial cellular mechanism that is completely independent of NMDA receptor modulation. While the effects of NV-5138 and ketamine converge on mTORC1 signaling, the actions of ketamine occur via a burst of glutamate and require AMPA receptor activation, based on evidence that pretreatment with an AMPA receptor antagonist (NBQX) blocks the antidepressant actions of ketamine as well as other rapid-acting agents $(7,10,12,39,40)$. Using the same dose as was used in these previous studies, we found that NBQX pretreatment had no effect on the antidepressant actions of NV-5138 in the FST and only a partial, nonsignificant blockade in NSFT. However, it is possible that a higher dose and/or additional doses of NBQX after NV-5138 could reveal a requirement for AMPA receptor activity.

Further studies will be needed to determine whether NV-5138 produces side effects in rodent models and in primates that overlap with or are different from the dissociative and psychotomimetic effects of ketamine. An obvious issue is whether NV-5138 activation of mTORC1 would influence tumor growth. While it is true that the chronic hyperactivation of mTORC1 induced either genetically or through natural mutations (e.g., TSC1/2 inactivation) has been associated with the development and/or propagation of certain cancers, this is quite different from the observed effects of NV-5138 on mTORC1 activation in the current context. Importantly, the pharmacokinetics of NV-5138 in rodents is relatively short ( $\sim 3$ hours) and the extent of mTORC1 activation is limited (2- to 3-fold) compared with genetically induced hyperactivation (6- to 8-fold) that has been associated with pathological etiologies of mTORC1 hyperaction (e.g., development of TSC (47). This is due to the self-limiting activation of mTORC1 by NV-5138 through the amino acid activation pathway due to the upstream regulation imparted by the GATOR2 complex (22). Thus, there is a low probability that treatment with NV-5138 will result in pathologies sometimes associated with mTORC1 pathway hyperactivation.

In conclusion, this is the first evidence, to our knowledge, that direct activation of mTORC1 can have positive effects in various models of antidepressant response and depressive behavior that are associated with changes in synaptic protein expression and morphology, directly confirming the critical role of mTORC1 activation for these processes. Characterization of this agent and mechanism for stimulation of mTORC1 signaling that causes synaptic and antidepressant behavioral response provides an alternative mechanistic approach for the treatment of depression.

\section{Methods}

Study design. Studies were primarily designed to determine the antidepressant-like efficacy and mechanism of action of NV-5138 in depression models. For in vivo behavioral studies, Western blot, and electrophysiology experiments, age-matched animals were randomly assigned to drug or vehicle treatment groups. The numbers of animals for each study type were determined on the basis of previous experience with the animal models utilized and based on results of pilot studies. Experiments were performed with at least 2 different batches of animals, and it was confirmed that both experiments show the same trend.

Animals and drug administration. Male Sprague-Dawley rats (Charles River Laboratories) weighing 250-260 g were used for most studies, with the exception of the CUS experiment, which used rats weighing 120-140 $\mathrm{g}$ because of the longer duration of the study. In addition, mutant BDNF Val66Met knockin mice (Val/Val WT, heterozygous Val/Met, and homozygous Met/Met mice at 8-12 weeks) generated as previously described were used to test the role of BDNF (33). Animals were singly housed and maintained in standard conditions with a 12-hour light/12-hour dark cycle and ad libitum access to food and water. Rats received a single oral administration of vehicle $(0.5 \%$ methylcellulose and 0.1\% Tween 80), NV-5138 (40, 80, or $160 \mathrm{mg} / \mathrm{kg}$, p.o., Navitor Pharmaceuticals Inc.), ketamine (10 mg/kg, i.p.), DMSO (0.5\%; $1 \mathrm{ml} / \mathrm{kg}$, i.p.), or NBQX (10 mg/kg, i.p., Tocris Bioscience). For the repeated dose study, NV-5138 (40 or $80 \mathrm{mg} / \mathrm{kg}$, p.o.) was administered daily for a total of 7 days and ketamine was injected $(10 \mathrm{mg} / \mathrm{kg}$, i.p.) every other day for 6 days.

Surgical and infusion procedures. Rats were anesthetized with 80 $\mathrm{mg}$ ketamine $/ 6 \mathrm{mg} / \mathrm{kg}$ xylazine i.p., and bilateral 22-gauge guide cannula were implanted at $0.5 \mathrm{~mm}$ above the site of infusion $(+3.0 \mathrm{~mm}$ $\mathrm{AP} ; \pm 1.0 \mathrm{~mm} \mathrm{ML} ;-4.0 \mathrm{~mm}$ DV to the bregma). In previous studies, we found that this anesthetic dose of ketamine did not produce an antidepressant response (7). Following 9 to 14 days of recovery, rats were bilaterally infused with one of a number of different agents, including the mTORC1 inhibitor rapamycin (Cell Signaling; $0.005 \mathrm{nmol} / \mathrm{side}$ or $10 \%$ DMSO vehicle for control), a function-blocking anti-BDNF Ab (Chemicon; $0.5 \mu \mathrm{g} /$ side, or normal sheep IgG for control; R\&D Systems) at a rate of $0.25 \mu \mathrm{l} / \mathrm{min}$ for 2 minutes. These procedures and doses have been validated in previous studies $(7,8,34,35)$. After the behavioral studies, brains were collected, coronal sections were cut in a cryostat, and cannula tip location was confirmed after Nissl staining.

CUS. CUS was carried out as previously described (8). Animals were exposed to a variable sequence of mild and unpredictable stressors for 21 days. A total of 13 different stressors were used ( 2 stressors per day). The stressors included rotation on a shaker, placement in a $4^{\circ} \mathrm{C}$ ambient temperature, lights off for 3 hours (10:00 AM-1:00 PM), lights on overnight, strobe light overnight, forced swim, $45^{\circ}$ tilted cages, food and water deprivation, crowded housing, wet bedding, white noise, restraint in plastic bag for 1 hour, and no bedding. The CUS protocol was continued until day 26 for subsequent behavioral testing and tissue sampling.

Behavior studies: FST. The FST was carried out as previously described for rat and mouse $(7,35,48)$. Each rat was subjected to a 15 -minute preswim in a Plexiglas cylinder $(30 \mathrm{~cm}$ diameter, $65 \mathrm{~cm}$ height) filled with water $\left(25 \pm 1^{\circ} \mathrm{C}, 45 \mathrm{~cm}\right.$ depth). After 24 hours, rats received drug treatments, and the next day ( 24 hours later), each rat was again placed in the swim cylinders for a 10-minute period and videotaped. Data were analyzed in a blinded manner by scoring the total immobility time during the entire 10-minute swim period. Each mouse was placed in a 41 glass beaker $(16 \mathrm{~cm}$ diameter, $24.5 \mathrm{~cm}$ height) containing water $\left(25 \pm 1^{\circ} \mathrm{C}, 15 \mathrm{~cm}\right.$ depth) for 10 minutes and videotaped. The duration of immobility was scored between 2 and 6 minutes by an experimenter blinded to the treatment groups.

NSFT. The NSFT was performed as previously described $(7,35)$. Briefly, animals were food deprived overnight and placed in an open 
field with a small amount of food in the center. The latency to feed was measured with a cut-off time of 15 minutes. After the NSFT, HCF during a 10-minute period was measured to verify motivation to feed.

FUST. The FUST was carried out as previously described (35, 49). Briefly, animals were habituated to a sterile cotton-tipped applicator placed into their home cage for 1 hour. Then each animal was exposed to a water-dipped cotton-tipped applicator for 5 minutes. After a 45-minute interval, each animal was exposed to a cottontipped applicator infused with fresh urine from females of the same strain for 5 minutes. The time spent sniffing the cotton-tipped applicator was measured during exposure to both water and urine by a blinded experimenter. Time spent biting the cotton-tip was excluded from the analysis.

Locomotor activity test. Animals were placed in a clean testing cage $(46 \mathrm{~cm} \times 23 \mathrm{~cm} \times 20 \mathrm{~cm}$ for rats, $30 \mathrm{~cm} \times 19 \mathrm{~cm} \times 13 \mathrm{~cm}$ for mice) for 30 minutes, during which time the number of beam breaks was measured using Med-PC software (Med Associates).

$S P T$. The SPT was carried out as previously described (8). Briefly, rats were exposed to a palatable sucrose solution (1\%; MilliporeSigma) for 48 hours, followed by 4 hours of water deprivation and 1 hour exposure to 2 identical bottles, one filled with sucrose solution and the other with water. Sucrose and water consumption were determined by measuring the change in the volume of fluid consumed. Sucrose preference was defined as the ratio of the volume of sucrose versus total volume of sucrose and water consumed during the 1-hour test.

Western blot. Tissue preparations and Western blots were conducted as previously described $(35,49)$. Olfactory bulbs were removed and $\mathrm{PFC}$ from adult rats was dissected from coronal sections of brain rostral to the corpus callosum. PFC samples were homogenized in a solution containing 0.32 M sucrose, 20 mM HEPES (pH 7.4), 1 mM EDTA, $1 \times$ protease inhibitor cocktail, $5 \mathrm{mM} \mathrm{NaF}$, and $1 \mathrm{mM}$ sodium vanadate. The homogenate was centrifuged for 10 minutes at $900 \mathrm{~g}$ at $4^{\circ} \mathrm{C}$, and the supernatant was centrifuged at $16,000 \mathrm{~g}$ for 10 minutes. After centrifugation, the supernatant (cytosolic fraction) was removed and the pellet (crude synaptosomal fraction) was resuspended by sonication in protein lysis buffer (50 mM Tris- $\mathrm{HCl}$ [pH 7.5], $150 \mathrm{mM} \mathrm{NaCl}, 1 \%$ Triton X-100, 0.1\% SDS, 2 mM EDTA, $1 \mathrm{mM} \mathrm{NaVO}_{3}, 5 \mathrm{mM} \mathrm{NaF}$, and $1 \times$ protease inhibitor cocktail). Protein concentration was determined by BCA protein assay. For Western blotting, equal amounts of protein (20 $\mu \mathrm{g})$ for each sample were loaded into $4 \%-20 \%$ SDS PAGE gel for electrophoresis. PVDF membranes with transferred proteins were blocked with $5 \%$ BSA or $5 \%$ skim milk in TBST (TBS + 0.1\% Tween-20) for 1 hour and kept with primary Abs overnight at $4^{\circ} \mathrm{C}$. The following primary Abs were used: phospho-mTOR (Ser2448, Cell Signaling, cata$\log 2971,1: 1000$ ), total mTOR (Cell Signaling, catalog 2972, 1:1000), phospho-ERK (Thr202/Tyr204, Cell Signaling, catalog 9101,1:1000), total ERK (Cell Signaling, catalog 9102, 1:1000), phospho-p70S6K (Thr389, Cell Signaling, catalog 9205, 1:1000), total p70S6K (Cell Signaling, catalog 9202, 1:1000), phospho-4EBP1 (Thr37/46, Cell Signaling, catalog 9459, 1:1000), PSD95 (Cell Signaling, catalog 2507, 1:1000), glutamate AMPA receptor 1(GluR1) (Cell Signaling, catalog 13185, 1:1000), Synapsin1 (Cell Signaling, catalog 6710, 1:1000), and synaptic vesicle protein 2A (SV2A) (EMD Millipore, catalog AB15224, 1:1000). The next day, blots were washed 3 times in TBST and incubated with horseradish peroxidase-conjugated anti-rabbit secondary $\mathrm{Ab}$ (1:5000 to 1:10000) for 1 hour. After a final 3 washes with TBST, bands were detected using ECL. The blots then were incubated in stripping buffer (Thermo Scientific) for 30 minutes at room temperature, followed by 3 washes with TBST. The stripped blots were kept in blocking solution for 1 hour and incubated with the primary Ab directed against total levels of the respective protein or GAPDH (Cell Signaling, catalog 5174, 1:1000) for loading control. Densitometric analysis of immunoreactivity for each protein was conducted using Image Lab (Bio-Rad). Immunoreactivity was normalized to saline-treated control group values for each protein.

Electrophysiology. Brain slices were prepared as previously described $(7,8,11,27)$. Briefly, 1 day after drug treatments, rats were anesthetized (chloral hydrate, $400 \mathrm{mg} / \mathrm{kg}$, i.p.) and brains removed and placed in ice-cold $\left(4^{\circ} \mathrm{C}\right)$ artificial cerebrospinal fluid (ACSF) in which sucrose $(252 \mathrm{mM}$ ) was substituted for $\mathrm{NaCl}$ (sucrose-ACSF). A block of tissue containing prefrontal cortex and coronal slices (400 $\mu \mathrm{m})$ was cut in sucrose-ACSF with an oscillating-blade tissue slicer. Slices were placed in a submerged recording chamber; bath temperature was then raised to $32^{\circ} \mathrm{C}$. Known concentrations of drugs in ACSF were applied through a stopcock arrangement $(\sim 4 \mathrm{ml} / \mathrm{min})$ to reach the slice within 7 to 10 seconds. The standard ACSF ( $\mathrm{pH} 7.35$ ), equilibrated with $95 \% \mathrm{O}_{2} / 5 \% \mathrm{CO}_{2}$, contained $128 \mathrm{mM} \mathrm{NaCl}, 3 \mathrm{mM} \mathrm{KCl}, 2 \mathrm{mM}$ $\mathrm{CaCl}_{2}, 2 \mathrm{mM} \mathrm{MgSO}_{4}, 24 \mathrm{mM} \mathrm{NaHCO}_{3}, 1.25 \mathrm{mM} \mathrm{NaH}_{2} \mathrm{PO}_{4}$, and $10 \mathrm{mM}$, $\mathrm{D}$-glucose. There was a recovery period of 1 to 2 hours before recording. Pyramidal neurons in layer $\mathrm{V}$ were visualized by videomicroscopy using a microscope ( $\times 40$ IR lens) with infrared differential interference contrast (IR/DIC). Patch pipettes (3-5 M $\Omega$ ) were pulled from glass tubing with a Flaming-Brown Horizontal Puller. The pipette solution contained the following: $115 \mathrm{mM} \mathrm{K}$ gluconate, $5 \mathrm{mM} \mathrm{KCl}, 2 \mathrm{mM}$ $\mathrm{MgCl}_{2}, 2 \mathrm{mM} \mathrm{Mg-ATP}, 2 \mathrm{mM} \mathrm{Na}{ }_{2} \mathrm{ATP}, 10 \mathrm{mM} \mathrm{Na}$-phosphocreatine, $0.4 \mathrm{mM} \mathrm{Na}_{2} \mathrm{GTP}$, and $10 \mathrm{mM}$ Hepes, $\mathrm{pH}$ 7.33. Neurobiotin (0.3\%) was added to the pipette solution to mark cells for later imaging. Wholecell recordings were made with an Axoclamp-2B amplifier. The output signal was low pass-filtered at $3 \mathrm{KHz}$ and digitized at $15 \mathrm{kHz}$; data were acquired by pClamp 10.2/Digidata 1550 A software. Series resistance, which was monitored throughout the experiment, was usually between 4 and $8 \mathrm{M} \Omega$. To minimize series-resistance errors, cells were discarded if series resistance rose above $10 \mathrm{M} \Omega$. Postsynaptic currents were studied in the continuous single-electrode voltage-clamp mode (3000 Hz low-pass filter) clamped $-65 \mathrm{mV}$ to separate the IPSCs from the EPSCs. After completion of recording, slices were transferred to $4 \%$ paraformaldehyde ( $0.1 \mathrm{M}$ phosphate buffer) and stored overnight at $4^{\circ} \mathrm{C}$. Slices were then processed with streptavidin conjugated to Alexa Fluor 594 (1:1000) for visualization of labeled cells.

Spine analysis. Labeled mPFC layer V neurons were imaged on a confocal laser scanning microscope (Olympus FV1000) equipped with a $\times 100,1.42$ NA objective at a zoom of $\times 5$ (voxel size $0.049 \mu \mathrm{m} \times$ $0.049 \mu \mathrm{m} \times 0.2 \mu \mathrm{m})$. Spine density was sampled at tips of tuft branches as they approach the pial membrane at a site approximately $100 \mu \mathrm{m}$ more proximal and midway between distal and proximal sites (length of dendritic segments was approximately $25 \mu \mathrm{m}$ ). Computerized analysis of z-stack images was performed on deconvolved confocal image stacks (AutoquantX Version 3.0.1, Media Cybernetics), and spines were and quantified using NeuronStudio software by an experimenter blinded to treatment history.

Statistics. Data for Western blot and behavioral studies were analyzed using 1-way or 2-way ANOVA, and differences between individual group means were then assessed by post hoc Tukey's multiple comparison test. Differences between group means for electrophysiol- 
ogy and spine analysis were assessed by Student's $t$ tests. Effects were considered significant at $P \leq 0.05$, and results are presented as mean \pm SEM. Analysis was conducted using GraphPad Prism 6 software.

Study approval. Animal use and procedures were in accordance with NIH guidelines and approved by the Yale University Animal Care and Use Committees.

\section{Author contributions}

TK designed the study, performed experiments and data analysis, and wrote the manuscript. SP performed experiments and data analysis and assisted in editing/revision of manuscript. RJL performed electrophysiological experiments and analyzed data. CHD performed spine analysis and analyzed data. RT performed Western blots. GPV, ES, SH, and RSD assisted with study design, data interpretation, and writing the manuscript.

\section{Acknowledgments}

This study was supported by research grants from Navitor Pharmaceuticals Inc., NIMH grants MH093897 and MH105910 (to RSD), and Sumitomo Dainippon Pharma (to TK).

Address correspondence to: Ronald S. Duman, 34 Park Street, New Haven, Connecticut 06519, USA. Phone: 203.974.7726; Email: ronald.duman@yale.edu.
1. Hasin DS, et al. Epidemiology of adult DSM-5 major depressive disorder and its specifiers in the United States. JAMA Psychiatry. 2018;75(4):336-346

2. Fava M. Diagnosis and definition of treatmentresistant depression. Biol Psychiatry. 2003;53(8):649-659.

3. Rush AJ, et al. Sequenced treatment alternatives to relieve depression $\left(\mathrm{STAR}^{*} \mathrm{D}\right)$ : rationale and design. Control Clin Trials. 2004;25(1):119-142.

4. Trivedi MH, et al. Evaluation of outcomes with citalopram for depression using measurementbased care in STAR ${ }^{*}$ D: implications for clinical practice. Am J Psychiatry. 2006;163(1):28-40.

5. Abdallah CG, Sanacora G, Duman RS, Krystal JH. Ketamine and rapid-acting antidepressants: a window into a new neurobiology for mood disorder therapeutics. Annu Rev Med. 2015;66:509-523.

6. Berman RM, et al. Antidepressant effects of ketamine in depressed patients. Biol Psychiatry. 2000;47(4):351-354.

7. Li N, et al. mTOR-dependent synapse formation underlies the rapid antidepressant effects of NMDA antagonists. Science. 2010;329(5994):959-964.

8. Li N, et al. Glutamate N-methyl-D-aspartate receptor antagonists rapidly reverse behavioral and synaptic deficits caused by chronic stress exposure. Biol Psychiatry. 2011;69(8):754-761.

9. Dwyer JM, Lepack AE, Duman RS. mTOR activation is required for the antidepressant effects of mGluR $/ 3$ blockade. Int J Neuropsychopharmacol. 2012;15(4):429-434.

10. Fukumoto K, et al. Activity-dependent brainderived neurotrophic factor signaling is required for the antidepressant actions of (2. Proc Natl Acad Sci U S A. 2019;116(1):297-302.

11. Liu RJ, et al. GLYX-13 produces rapid antidepressant responses with key synaptic and behavioral effects distinct from ketamine. Neuropsychopharmacology. 2017;42(6):1231-1242.

12. Voleti B, et al. Scopolamine rapidly increases mammalian target of rapamycin complex 1 signaling, synaptogenesis, and antidepressant behavioral responses. Biol Psychiatry. 2013;74(10):742-749.

13. Dwyer JM, Maldonado-Avilés JG, Lepack AE, DiLeone RJ, Duman RS. Ribosomal protein S6 kinase 1 signaling in prefrontal cortex controls depressive behavior. Proc Natl Acad Sci U S A. 2015;112(19):6188-6193.

14. Zoncu R, Bar-Peled L, Efeyan A, Wang S, Sancak
Y, Sabatini DM. mTORC1 senses lysosomal amino acids through an inside-out mechanism that requires the vacuolar $\mathrm{H}(+)$-ATPase. Science. 2011;334(6056):678-683.

15. Dibble CC, Manning BD. Signal integration by mTORC1 coordinates nutrient input with biosynthetic output. Nat Cell Biol. 2013;15(6):555-564.

16. Cota D, et al. Hypothalamic mTOR signaling regulates food intake. Science. 2006;312(5775):927-930.

17. Hara K, Yonezawa K, Weng QP, Kozlowski MT, Belham C, Avruch J. Amino acid sufficiency and mTOR regulate p70 S6 kinase and eIF-4E BP1 through a common effector mechanism. J Biol Chem. 1998;273(23):14484-14494.

18. Fox HL, Pham PT, Kimball SR, Jefferson LS, Lynch CJ. Amino acid effects on translational repressor 4E-BP1 are mediated primarily by L-leucine in isolated adipocytes. Am J Physiol. 1998;275(5):C1232-C1238.

19. Lynch CJ, Fox HL, Vary TC, Jefferson LS, Kimbal SR. Regulation of amino acid-sensitive TOR signaling by leucine analogues in adipocytes. J Cell Biochem. 2000;77(2):234-251.

20. Efeyan A, Comb WC, Sabatini DM. Nutrientsensing mechanisms and pathways. Nature. 2015;517(7534):302-310.

21. Saxton RA, et al. Structural basis for leucine sensing by the Sestrin2-mTORC1 pathway. Science. 2016;351(6268):53-58.

22. Sengupta S, et al. Discovery of NV-5138, the first selective Brain mTORC1 activator. Sci Rep. 2019;9(1):4107.

23. Sakai R, Cohen DM, Henry JF, Burrin DG, Reeds PJ. Leucine-nitrogen metabolism in the brain of conscious rats: its role as a nitrogen carrier in glutamate synthesis in glial and neuronal metabolic compartments. JNeurochem. 2004;88(3):612-622.

24. Liu RJ, Fuchikami M, Dwyer JM, Lepack AE, Duman RS, Aghajanian GK. GSK-3 inhibition potentiates the synaptogenic and antidepressantlike effects of subthreshold doses of ketamine. Neuropsychopharmacology. 2013;38(11):2268-2277.

25 . Willner P. Validity, reliability and utility of the chronic mild stress model of depression: a 10-year review and evaluation. Psychopharmacology (Berl). 1997;134(4):319-329.

26. Willner P. Chronic mild stress (CMS) revisited: consistency and behavioural-neurobiological concordance in the effects of CMS. Neuropsychobiology. 2005;52(2):90-110.

27. Liu RJ, Aghajanian GK. Stress blunts serotonin- and hypocretin-evoked EPSCs in prefrontal cortex: role of corticosterone-mediated apical dendritic atrophy. Proc Natl Acad Sci US A. 2008;105(1):359-364.

28. Liston C, et al. Stress-induced alterations in prefrontal cortical dendritic morphology predict selective impairments in perceptual attentional set-shifting. J Neurosci. 2006;26(30):7870-7874

29. Radley JJ, Rocher AB, Janssen WG, Hof PR, McEwen BS, Morrison JH. Reversibility of apical dendritic retraction in the rat medial prefrontal cortex following repeated stress. Exp Neurol. 2005;196(1):199-203.

30. Radley JJ, et al. Repeated stress induces dendritic spine loss in the rat medial prefrontal cortex. Cereb Cortex. 2006;16(3):313-320.

31. Radley JJ, et al. Repeated stress alters dendritic spine morphology in the rat medial prefrontal cortex. J Comp Neurol. 2008;507(1):1141-1150.

32. Joëls M, et al. Effects of chronic stress on structure and cell function in rat hippocampus and hypothalamus. Stress. 2004;7(4):221-231.

33. Chen ZY, et al. Genetic variant BDNF (Val66Met) polymorphism alters anxiety-related behavior. Science. 2006;314(5796):140-143.

34. Lepack AE, Fuchikami M, Dwyer JM, Banasr $\mathrm{M}$, Duman RS. BDNF release is required for the behavioral actions of ketamine. Int J Neuropsychopharmacol. 2014;18(1):null.

35. Kato T, Fogaça MV, Deyama S, Li XY, Fukumoto $\mathrm{K}$, Duman RS. BDNF release and signaling are required for the antidepressant actions of GLYX-13. Mol Psychiatry. 2018;23(10):2007-2017.

36. Autry AE, et al. NMDA receptor blockade at rest triggers rapid behavioural antidepressant responses. Nature. 2011;475(7354):91-95.

37. Liu RJ, Lee FS, Li XY, Bambico F, Duman RS, Aghajanian GK. Brain-derived neurotrophic factor Val66Met allele impairs basal and ketamine-stimulated synaptogenesis in prefrontal cortex. Biol Psychiatry. 2012;71(11):996-1005.

38. Ghosal S, et al. Activity-dependent brain-derived neurotrophic factor release is required for the rapid antidepressant actions of scopolamine. Biol Psychiatry. 2018;83(1):29-37.

39. Maeng S, et al. Cellular mechanisms underlying the antidepressant effects of ketamine: role of alpha-amino-3-hydroxy-5-methylisoxazole4-propionic acid receptors. Biol Psychiatry. 2008;63(4):349-352.

40. Burgdorf J, et al. GLYX-13, a NMDA receptor glycine-site functional partial agonist, induces antidepressant-like effects without ketamine-like side effects. Neuropsychopharmacology. 
2013;38(5):729-742.

41. Sánchez C, Meier E. Behavioral profiles of SSRIs in animal models of depression, anxiety and aggression. Are they all alike? Psychopharmacology (Berl). 1997;129(3):197-205.

42. Bodnoff SR, Suranyi-Cadotte B, Aitken DH, Quirion R, Meaney MJ. The effects of chronic antidepressant treatment in an animal model of anxiety. Psychopharmacology (Berl). 1988;95(3):298-302.

43. Bodnoff SR, Suranyi-Cadotte B, Quirion R, Meaney MJ. A comparison of the effects of diazepam versus several typical and atypical anti-depressant drugs in an animal model of anxiety. Psychopharmacology (Berl). 1989;97(2):277-279.

44. Papp M, Nalepa I, Antkiewicz-Michaluk L, Sánchez C. Behavioural and biochemical studies of citalopram and WAY 100635 in rat chronic mild stress model. Pharmacol Biochem Behav. 2002;72(1-2):465-474.

45. Abdallah CG, Sanacora G, Duman RS, Krystal JH. Ketamine and rapid-acting antidepressants: a window into a new neurobiology for mood disorder therapeutics. Annu Rev Med. 2015;66:509-523.

46. Navarria A, et al. Rapid antidepressant actions of scopolamine: Role of medial prefrontal cortex and M1-subtype muscarinic acetylcholine recep- tors. Neurobiol Dis. 2015;82:254-261.

47. Zordan P, Cominelli M, Cascino F, Tratta E, Poliani PL, Galli R. Tuberous sclerosis complexassociated CNS abnormalities depend on hyperactivation of mTORC1 and Akt. JClin Invest. 2018;128(4):1688-1706.

48. Ota KT, et al. REDD1 is essential for stressinduced synaptic loss and depressive behavior. Nat Med. 2014;20(5):531-535.

49. Dutheil S, Ota KT, Wohleb ES, Rasmussen K, Duman RS. High-fat diet induced anxiety and anhedonia: impact on brain homeostasis and inflammation. Neuropsychopharmacology. 2016;41(7):1874-1887. 